\title{
Immunohistochemical staining of plastic embedded bone marrow trephine biopsy specimens after microwave heating
}

W G McCluggage, S Roddy, C Whiteside, J Burton, H McBride, P Maxwell, H Bharucha

\begin{abstract}
Aims-To investigate (1) whether adequate immunohistochemical staining can be achieved on sections cut from plastic embedded bone marrow trephine biopsy specimens after microwave heating in citrate buffer; and (2) whether this immunohistochemical staining is comparable with that achieved on routine sections cut from paraffin wax embedded trephine biopsy specimens after decalcification procedures.
\end{abstract}

Methods-Sixty five consecutive bone marrow trephine biopsy specimens of more than $1 \mathrm{~cm}$ in length were divided transversely into two equal parts. One part was processed in paraffin wax followed by decalcification. The other part was embedded in the epoxyresin Polarbed 812 followed by the cutting of $1 \mu \mathrm{m}$ sections. Both parts underwent immunohistochemical staining by an identical panel of antibodies. With Polarbed 812 plastic embedded sections, microwave heating in citrate buffer was undertaken before the application of antisera.

Results-On sections cut from plastic embedded material, immunohistochemical staining was generally satisfactory, easy to interpret and comparable with that achieved with paraffin wax embedded material. Exceptions were antibodies to neutrophil elastase and CD61 where immunostaining was consistently negative on plastic embedded sections. Immunohistochemical staining for CD20 was consistently more reliable on plastic embedded sections.

Conclusions-The results provide evidence that, with few exceptions, satisfactory immunohistochemical staining is possible on plastic embedded bone marrow trephine biopsy specimens after microwave heating in citrate buffer. This, combined with the advantage of superior cellular morphology with semi-thin $(1 \mu \mathrm{m})$ sections of plastic embedded material, make such embedding procedures the preferred method for the processing of bone marrow trephine biopsy specimens.

(f Clin Pathol 1995;48:840-844)

Keywords: Bone marrow trephine biopsy specimen immunohistochemistry, plastic embedding, microwave heating.
Bone marrow trephine biopsies are routinely performed, in conjunction with marrow aspirates, in the investigation of patients with a variety of haemopoietic disorders, both neoplastic and non-neoplastic. Trephine biopsy specimens are particularly useful where there is significant bone marrow fibrosis which frequently results in an inadequately aspirated specimen or "dry tap". Trephine biopsy specimens are also useful in the assessment of marrow cellularity and in the determination of the extent of marrow involvement by neoplastic or other infiltrates. The relation between cellular elements and normal marrow structures, such as bony trabeculae and blood vessels, can be assessed accurately only on bone marrow trephine biopsy specimens. In addition focal lesions, such as granulomas, are more readily identifiable on trephine biopsy specimens than on marrow aspirates.

Two main methods are used for the routine histological examination of bone marrow trephine biopsy specimens, namely paraffin wax embedding followed by decalcification and plastic embedding followed by the cutting of $1 \mu \mathrm{m}$ sections. A third approach to processing such specimens is the use of cryostat sections of undecalcified bone marrow biopsy specimens. This method, although allowing for optimal antigen preservation, results in very poor cellular morphology, a serious obstacle in evaluating these specimens, and thus has not gained widespread acceptance. Problems arise in cutting sections of bone marrow trephine biopsy specimens because of the intimate mixture of hard tissue (bone) and soft tissue (marrow and fat). To cut adequate intact sections, one can either make the tissue uniformly soft by paraffin wax embedding followed by decalcification, or make the tissue uniformly hard by using a plastic embedding procedure. There has been much debate in the literature regarding the relative merits of these two techniques. ${ }^{1-7}$ Those who advocate paraffin wax embedding of trephine biopsy specimens stress the advantages of the familiarity of most pathologists with sections cut from such material combined with their unfamiliarity with plastic embedded sections and the fact that widespread antigen preservation allows a wide range of immunohistochemical reactions to be performed. ${ }^{12}$ Furthermore, plastic embedding represents a significant departure from normal routine for the diagnostic histopathology laboratory as special equipment, different preparation protocols 
and a higher degree of technical expertise are required. The protagonists of plastic embedding of bone marrow trephine biopsy specimens point to the advantages of the excellent cellular morphology due to the thin sections obtained by this technique and to the lack of tissue distortion which is inevitable following decalcification procedures. ${ }^{36}$ In addition, plastic embedding procedures allow a wide range of histochemical stains to be performed. ${ }^{4} \mathrm{~A}$ major disadvantage with plastic embedded material is the loss of immunoreactivity which has hampered the routine application of immunohistochemical reactions in the evaluation of such trephine biopsy specimens.

Two main types of plastic resin are available for embedding tissue, namely epoxy and acrylic resins. ${ }^{8}$ Acrylic resins include methyl methacrylate and glycol methacrylate. In recent years glycol methacrylate has become the resin of choice for embedding bone marrow trephine biopsy specimens in plastic. ${ }^{45}$ Several authors have described methods of glycol methacrylate embedding which combine the advantage of excellent cellular morphology with optimal antigen preservation, permitting the immunohistochemical detection of a wide range of cellular antigens. ${ }^{57}$ We routinely use the epoxyresin Polarbed 812 for processing bone marrow trephine biopsy specimens. This resin is also used for the preparation of semi-thin tissue sections prior to the cutting of ultrathin sections for routine electron microscopy examination. In utilising this resin for the preparation of bone marrow trephine biopsy specimens we have avoided the need for specialised procedures and equipment. Previously we have had disappointing results when attempting to perform immunohistochemical reactions on tissue embedded in this resin, with little or no specific staining together with excessive background staining. Several articles have recently appeared in the literature advocating the use of prior microwave heating to enhance immunohistochemical staining on a variety of tissue types. ${ }^{9-14}$ These reports stimulated us to undertake this study to determine whether prior microwave heating would allow a range of antigens to be detected, using routine immunohistochemical techniques, in sections cut from plastic embedded tissue. We have compared results of immunohistochemical staining between routine paraffin wax embedded material without microwave heating and plastic embedded material following microwave heating in citrate buffer.

\section{Methods}

Sixty five consecutive bone marrow trephine biopsy specimens more than $1 \mathrm{~cm}$ in length were studied. All were obtained from the posterior, superior iliac crest of patients seen by a haematologist at the Royal Group of Hospitals, Belfast, or the Mater Infirmorum Hospital, Belfast. Trephine biopsy specimens were received fresh and processed in the Department of Pathology, Royal Group of Hospitals, Belfast.
FIXATION AND PROCESSING OF TREPHINE BIOPSY SPECIMENS

All specimens were divided transversely into two equal parts. One part was fixed in 3\% acetic acid in $10 \%$ unbuffered formol saline for a minimum of one and a half hours and a maximum of three hours. It was then washed in running water, treated with EDTA in buffer (pH 7.0) for 24 hours and processed to paraffin wax. Sections $(5 \mu \mathrm{m})$ were cut and stained with haematoxylin and eosin, and Giemsa.

The second part of each trephine biopsy was fixed overnight in $10 \%$ neutral buffered formalin, followed by washing in phosphate buffer prior to processing. Processing was carried out on a rotator as follows: $50 \%$ alcohol (30 minutes), $70 \%$ alcohol ( 30 minutes), $90 \%$ alcohol (30 minutes), $100 \%$ alcohol $(2 \times 30$ minutes), dried acetone ( $2 \times 30$ minutes $), 1$ acetone: 3 resin (one hour), neat resin at $37^{\circ} \mathrm{C}$ ( $2 \times 1.5$ hours). The trephine biopsy specimen was then embedded in a rubber mould or plastic capsule and polymerised at $60^{\circ} \mathrm{C}$ overnight. Blocks were trimmed on a Leica Ultracut $\mathrm{E}$ Ultramicrotome, set at $1 \mu \mathrm{m}$, using a $45^{\circ}$ glass knife. Sections $(1 \mu \mathrm{m})$ were then cut using a Histo-Diatome diamond knife. Each section was lifted onto a slide containing a drop of water, flamed to remove any wrinkles, dried on a hot plate and reflamed to ensure the section did not wash off during staining. Etching of sections was undertaken prior to staining. Slides were placed in a jar containing sodium ethoxide for 25 minutes, washed in $50 \%$ alcohol for 30 minutes and then in running water for 15 minutes. Sections were stained with haemotoxylin and eosin, and Giemsa.

The resin used for this plastic embedding procedure was the epoxyresin Polarbed 812 (Fison's Scientific Equipment, Loughborough, UK). This was made up as follows: Polarbed 812 (50 g), DDSA (32 g), MNA (21 g), and DMP-30 (2 g).

\section{IMMUNOHISTOCHEMICAL METHODS}

A number of primary antisera was applied to serial sections cut from both paraffin wax and plastic embedded tissue. An identical panel of antibodies was used to stain the two halves of each trephine biopsy specimen to facilitate comparison of immunohistochemical staining between sections cut from paraffin wax and plastic embedded tissue.

Sections cut from plastic embedded tissue were transferred onto APES coated slides (Sigma, Poole, Dorset, UK), etched in sodium ethoxide (25 minutes), rinsed in alcohol, endogenous peroxidase activity blocked in 3\% alcoholic hydrogen peroxide (15 minutes), and washed well in water. Slides were placed in a plastic Coplin jar filled with citrate buffer $(2 \cdot 1 \mathrm{~g}$ citric acid monohydrate in 1 litre of distilled water, $\mathrm{pH} 6.0$ ) and placed for 30 minutes in an $850 \mathrm{~W}$ domestic microwave oven. The Coplin jar was topped up at intervals with distilled water to avoid complete evaporation. After microwave heating slides were allowed to cool to room temperature. Incubation with the primary antibody was performed and antigens 
Antibodies used in the study together with their source and major specificities

\begin{tabular}{|c|c|c|}
\hline Antibody & Source & Main specificity \\
\hline $\begin{array}{l}\text { Leukocyte common antigen (LCA) (CD45) } \\
\text { L26 (CD20) } \\
\text { UCHL1 (CD45 RO) } \\
\text { MT1 (CD43) }\end{array}$ & $\begin{array}{l}\text { Dako, Copenhagen, Denmark } \\
\text { Dako } \\
\text { Dako } \\
\text { Biotest, Dreieich, Germany }\end{array}$ & $\begin{array}{l}\text { Leucocytes } \\
\text { B lymphoid cells } \\
\text { T lymphoid cells } \\
\text { T lymphoid cells, myeloid cells, megakaryocytes, } \\
\text { erythroid cells }\end{array}$ \\
\hline CD3 & Dako & T lymphoid cells \\
\hline CD61 & Dako & Megakaryocytes \\
\hline CD68 & Dako & Histiocytic cells, myeloid cells \\
\hline Factor VIII related antigen & Signet, St Louis, MO, USA & Megakaryocytes, endothelium \\
\hline Neutrophil elastase & Dako & Neutrophils \\
\hline Glycophorin A & $\begin{array}{l}\text { Mason DY, Nuffield Dept. of Haematology, John } \\
\text { Radcliffe Hospital, Oxford, UK }\end{array}$ & Erythroid cells \\
\hline Ber-H2 (CD30) & Dako & $\begin{array}{l}\text { Reed-Sternberg cells in Hodgkin's disease, activated } \\
\text { lymphoid cells, some high grade non-Hodgkin's } \\
\text { lymphomas }\end{array}$ \\
\hline Leu M1 (CD 15) & Dako & $\begin{array}{l}\text { Reed-Sternberg cells in Hodgkin's disease, } \\
\text { myelomonocytic cells, some adenocarcinomas }\end{array}$ \\
\hline Ki67 (MIB1) & The Binding Site, Birmingham, UK & Ki67 antigen \\
\hline Neurone specific enolase (NSE) & IncStar, Wokingham, UK & $\begin{array}{l}\text { Neuroendocrine cells and tumours, neural cells and } \\
\text { tumours }\end{array}$ \\
\hline $\begin{array}{l}\text { Cam } 5 \cdot 2 \\
\text { AE1/AE3 }\end{array}$ & $\begin{array}{l}\text { Becton Dickinson, Sunnyvale, CA, USA } \\
\text { ICN Biomedicals Ltd, Thame, UK }\end{array}$ & $\begin{array}{l}\text { Cytokeratins } \\
\text { Cytokeratins }\end{array}$ \\
\hline Carcinoembryonic antigen (CEA) & Dako & Adenocarcinomas, other carcinomas \\
\hline Epithelial membrane antigen (EMA) & Dako & Epithelial cells, plasma cells, some lymphomas \\
\hline Chromogranin A & Dako & Neuroendocrine cells and tumours \\
\hline S100 protein & Diagnostic Products Ltd, Abingdon, UK & $\begin{array}{l}\text { S100 protein (melanocytic lesions, dendritic cells, } \\
\text { others) }\end{array}$ \\
\hline $\begin{array}{l}\kappa \text { light chains } \\
\lambda \text { light chains }\end{array}$ & $\begin{array}{l}\text { Dako } \\
\text { Dako }\end{array}$ & $\begin{array}{l}\kappa \text { light chains } \\
\lambda \text { light chains }\end{array}$ \\
\hline
\end{tabular}

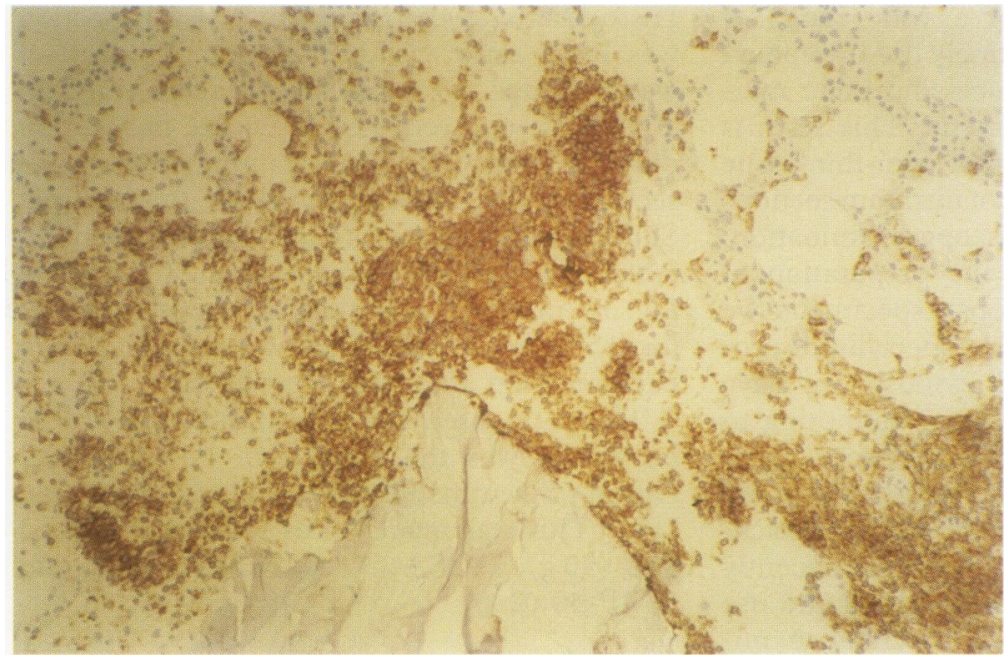

Figure 1 Immunohistochemistry of a plastic embedded bone marrow trephine specimen for CD20 (L26). (Case of low grade non-Hodgkin's lymphoma.)

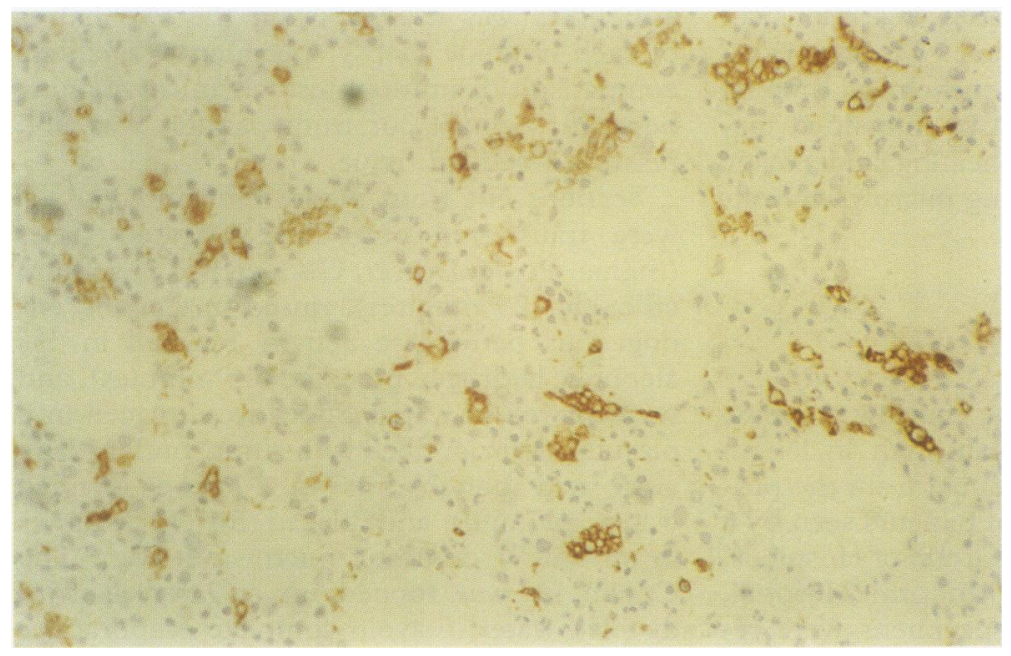

Figure 2 Immunohistochemistry of a plastic embedded bone marrow trephine specimen for CD68. (Normal bone marrow showing positive staining of myeloid cells.) were localised using a standard Streptavidinbiotin peroxidase method (Dako, Copenhagen, Denmark).

Sections from paraffin wax embedded tissue for immunohistochemistry were cut onto APES coated slides and dried at $56^{\circ} \mathrm{C}$ overnight. Immunohistochemical staining was as outlined for plastic embedded material, except sections were not subjected to prior microwave heating. Several antisera required pretreatment with trypsin (ICN, Amsterdam, Netherlands), namely $\mathrm{CD} 3, \mathrm{CD} 68, \kappa$ and $\lambda$ light chains, carcinoembryonic antigen (CEA), Cam $5 \cdot 2$, and AE1/AE3.

ANTIBODIES USED IN STUDY

The table shows the primary antisera used in the study together with their main specificities. These antibodies were chosen, some because they stain normal haemopoietic elements present in bone marrow and others because they are of use in the diagnosis of a variety of haemopoietic and non-haemopoietic disorders affecting the bone marrow.

\section{Results}

In all cases cellular morphology was superior with sections cut from plastic embedded tissue compared with those cut from paraffin wax embedded tissue. Microwave heating resulted in specific, easily interpretable staining of plastic embedded material with 20 out of 22 antibodies used in the study. Staining was comparable with that achieved with paraffin wax embedded sections (in which specific, easily interpretable staining was achieved without microwave heating with all 22 antibodies), although generally of lower intensity. Excessive background staining was not present. The two exceptions were antibodies against neutrophil elastase and CD61 where immunohistochemical staining was consistently negative on 


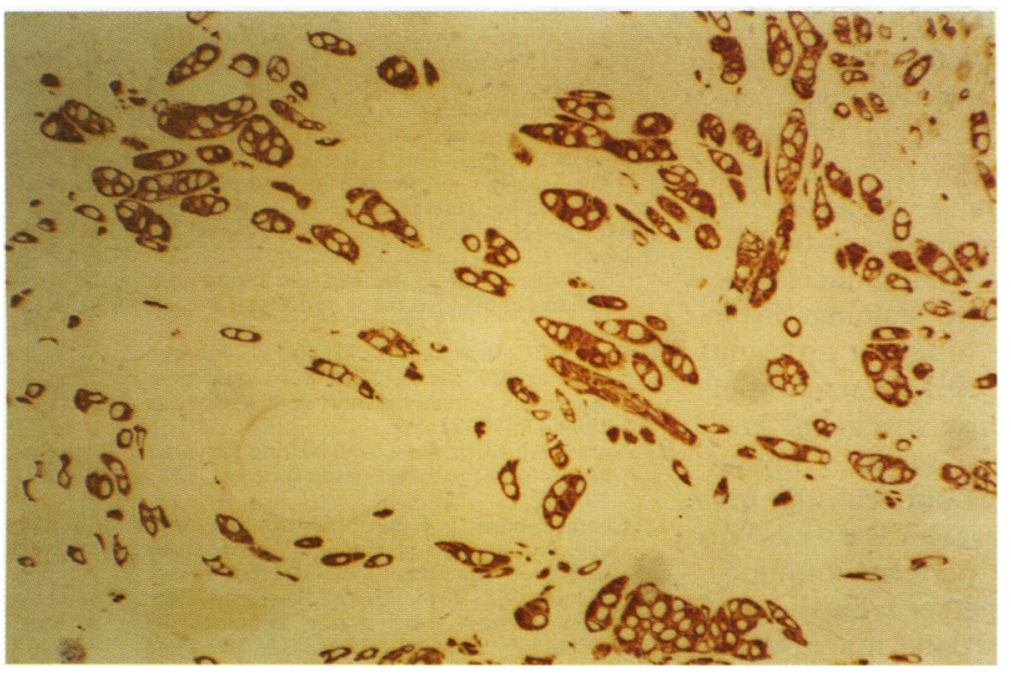

Figure 3 Immunohistochemistry of a plastic embedded bone marrow trephine biopsy specimen for Cam 5·2. (Case of metastatic breast carcinoma.)

sections cut from plastic embedded tissue but positive on sections cut from paraffin wax embedded tissue. Immunostaining with the CD20 antibody was more satisfactory on plastic embedded material. With all other antibodies used, immunostaining was comparable on sections cut from plastic and paraffin wax embedded material.

With sections cut from plastic embedded tissue and stained with neurone specific enolase (NSE), strong cytoplasmic staining of mature plasma cells was identified in a number of cases. This phenomenon was not seen in sections of paraffin wax embedded material stained with NSE.

Figures 1 to 3 show sections cut from plastic embedded biopsy specimens and stained with the antibodies CD20, CD68 and Cam 5.2.

\section{Discussion}

Immunohistochemical staining of tissue sections has become a valuable diagnostic tool for the histopathologist. With plastic embedded bone marrow trephine biopsy specimens, it has generally been difficult to achieve adequate immunohistochemical staining and, in most pathology laboratories, trephine biopsy specimens are routinely processed in paraffin wax. This is in spite of the superior cellular morphology obtainable with semi-thin sections cut from plastic embedded material. For plastic embedding of bone marrow trephine biopsy specimens, the water miscible plastic glycol methacrylate has become the resin of choice and, in recent years, there have been reports in the literature of modified glycol methacylate embedding techniques for bone marrow trephine biopsy specimens which permit high quality visualisation of morphological detail together with optimal antigen preservation. ${ }^{57}$ Such embedding techniques also allow a wide range of detailed enzyme histochemical reactions to be performed. ${ }^{15-17}$

In our laboratory we routinely use a plastic embedding technique for the histological examination of bone marrow trephine biopsy specimens. We use the epoxyresin Polarbed 812 , which was chosen primarily because it is also routinely used for electron microscopy. All trephine biopsy specimens are routinely processed in the Electron Microscopy Unit. This alleviates the need for the separate specimen preparation protocols and different equipment necessary if a different method of plastic embedding were to be used. Excellent cytological detail is achieved and the application of a range of enzyme histochemical reactions is possible. Ultrathin sections from the same block can also subsequently be examined by electron microscopy in cases where this is indicated.

In the past we have attempted immunohistochemical staining of such plastic embedded trephine biopsy specimens with little success. We found a lack of specific, readily interpretable staining together with excessive background, non-specific staining. With the recent interest in microwave heating, ${ }^{9-14}$ as opposed to the predigestion of tissue sections by proteolytic enzymes, as an easily applicable method for re-exposing antigens for immunohistochemical reactions, we undertook this study to investigate whether microwave heating would result in enhanced immunohistochemical staining on sections cut from plastic embedded bone marrow trephine biopsy specimens. The results provide evidence that prior microwave heating does indeed result in enhanced immunoreactivity with specific, readily interpretable staining and abolition of excessive background staining with most of antibodies used in the study. Staining was comparable with that achieved on paraffin wax embedded material without prior microwave heating. Only with antibodies against neutrophil elastase and CD61 was positive immunostaining not obtained. Although staining intensity was generally less with plastic than with paraffin wax embedded material, staining was specific; the less intense staining can be explained on the basis of the thinness $(1 \mu \mathrm{m})$ of sections obtained with the plastic embedding technique.

The exact mechanisms of antigen retrieval achieved following microwave heating are not established but it is possible that microwaves disrupt the cross-linking of proteins in a similar manner to enzymatic predigestion. ${ }^{18}$ An important consideration when undertaking microwave heating is whether normally masked antigens may be exposed, resulting in false positive results with previously trusted antibodies. ${ }^{19}$ The only unexpected binding pattern we have identified in the present study is positive cytoplasmic staining of plasma cells by NSE on plastic embedded material. Positive staining of plasma cells for NSE was not seen in sections cut from paraffin wax embedded material. This unexpected immunohistochemical stainingreaction was potentially misleading in bone marrow trephine biopsy specimens from two patients with neuroblastoma. A diagnosis of bone marrow involvement by metastatic neuroblastoma could have been rendered and inappropriate treatment instigated had it not been appreciated that the 
small numbers of cells exhibiting positive staining for NSE were, in fact, mature plasma cells.

In conclusion, the results of the study confirm that prior microwave heating results in unmasking of antigens and permits adequate immunohistochemical staining of plastic embedded material with a range of antibodies. Only with occasional antibodies did microwave heating not result in positive staining. Prior microwave heating may be of use in exposing antigens for immunohistochemical staining of bone marrow trephine biopsy specimens embedded in a variety of plastic media. Only by undertaking a study such as ours can the use of microwave antigen retrieval be assessed. We believe that the ability to perform immunohistochemical reactions together with the undoubted superior cytological detail make plastic embedding the method of choice for processing bone marrow trephine biopsy specimens. The study adds to the growing list of tissue types on which prior microwave heating results in improved immunohistochemical staining.

1 Gatter KC, Heryet A, Brown DC, Mason DY. Is it necessary to embed bone marrow biopsies in plastic for haematological diagnosis? Histopathology 1987;11:1-7.

2 Vincic L, Weston S, Ridell RH. Bone core biopsies. Plastic or paraffin? Am 7 Surg Pathol 1989;13:329-34.

3 Islam A, Frisch B. Plastic embedding in routine histology I: Preparation of semi-thin sections of undecalcified marrow cores. Histopathology 1985;9:1263-74.

4 Islam A, Henderson ES. Glycol methacrylate embedding for light microscopy I: Enzyme histochemistry of semithin sections of undecalcified marrow cores. $₹$ Clin Pathol 1987;40:1194-200.

5 Islam A, Archimbaud E, Henderson ES, Han T. Glycol methacrylate embedding for light microscopy II: Im- munohistochemical analysis of semi-thin sections of undecalcified marrow cores. 7 Clin Pathol 1988;41:892-6.

6 Casey TT, Cousar JB, Collins RD. A simplified plastic embedding and immunohistologic technique for immunophenotypic analysis of human haematopoietic and lymphoid tissues. Am f Pathol 1988;31:183-9.

7 Burgio VL, Pignoloni P, Baroni CD. Immunohistology of bone marrow: a modified method of glycol-methacrylate embedding. Histopathology 1991;18:37-43.

8 Murray GI. Is wax on the wane? $\mathcal{F}$ Pathol 1988;56:187-8.

9 Cattoretti G, Pileri S, Parravicini C, Becker MHG, Poggi $\mathrm{S}$, Bifulco $\mathrm{C}$, et al. Antigen unmasking on formalin-fixed, paraffin-embedded tissue sections. F Pathol 1993;171:8398.

10 Cuevas EC, Bateman AC, Wilkins BS, Johnson PA, Williams $\mathrm{JH}$, Lee AHS, et al. Microwave antigen retrieval in immunocytochemistry: a study of 80 antibodies. $₹$ Clin Pathol 1994;47:448-52.

11 Norton AJ. Microwave oven heating for antigen unmasking in routinely processed tissue sections. $\mathcal{F}$ Pathol 1993;171: $79-80$.

12 Charalambous C, Singh N, Isaacson PG. Immuno-histochemical analysis of Hodgkin's disease using microwave heating. F Clin Pathol 1993;46:1085-8.

13 Reynolds GM, Rowlands DC, Young JA. The use of microwave antigen retrieval for immunostaining in cytopathology [abstract]. F Pathol 1994;173:192.

14 Gerdes J, Becker MHG, Key G, Cattoretti G. Immunohistological detection of tumour growth fraction $(\mathrm{Ki}-$ 67 antigen) in formalin-fixed and routinely processed tissues. \& Pathol 1992;168:85-7.

15 Burgio VL, Morra E, Ascari E. Histochemistry on glycolmethacrylate embedded human bone marrow biopsies. A methacrylate embedded human bone marrow biopsies. A 177-87.

16 Westen H, Bainton DF. Association of alkaline-phosphatasepositive reticulum cells in bone marrow with granulocytic precursors. Ұ Exp Med 1979;150:919-37.

17 Beckstead JH, Halverson PS, Ries CA, Bainton DF. Enzyme histochemistry and immunohistochemistry on biopsy specimens of pathologic bone marrow. Blood 1981;57: 1088-98.

18 Shi SR, Key ME, Kalra KL. Antigen retrieval in formalinfixed, paraffin-embedded tissues: An enhancement method for immunohistochemical staining based on microwave oven heating of tissue sections. $\mathscr{f}$ Histochem $\mathrm{Cy}$ tochem 1991;39:741-8.

19 McKee PH, Hobbs C, Hall PA. Antigen retrieval by microwave irradiation lowers immunohistological detection thresholds. Histopathology 1993;23:377-9. 